\title{
An Overview: Semantic Web based Education
}

\author{
Lincy M Mathews \\ M S Ramaiah Institute of \\ Technology \\ Bangalore, Karnataka
}

\author{
Dr B R K Rao \\ Vellore Institute of Technology \\ Vellore University \\ Tamil Nadu
}

\begin{abstract}
The present educational systems are trying to incorporate the semantic web technologies, so as to provide an adaptable, personalized and an intelligent learning environment. The long term vision of Education is to provide AAAL: Anytime, Anywhere, Anybody Learning. This paper discusses the architecture of semantic web for the purpose education system which satisfies AAAL .It contains an overview of how ontology can represent the knowledge base which forms the backbone of the Semantic Web based Educational system, thus making the system useful to the learners. It also contains methods on how ontology construction can take place for a particular domain.
\end{abstract}

\section{General Terms}

Semantic Web, Ontology, Education System

\section{Keywords}

Education, Domain Ontology, Semantic Web Based Education System

\section{INTRODUCTION}

Semantic web applications are being developed in many areas and disciplines including education. For the intellectual development of human mind, both formal and informal education is important. In whichever format, education is being provided; the ability for the humans to learn effectively is of importance. For this, educationists try focusing on learning oriented rather than on teaching oriented.

Semantic Web based Educational Systems (SWEBS) is the name given by the AI in Education community to the new generation of such systems that use semantic web technologies to generate educational systems that are more personalized, adaptable and intelligent. The main goal is to use resources available on the Web through standard based technologies in order to accomplish AAAL: Anytime, Anywhere, Anybody Learning.

The Educational Semantic Web [5] is mainly based on three fundamental affordances. The first is the capacity for effective information storage and retrieval. The second is the capacity for nonhuman autonomous agents to augment the learning, information retrieval and processing power of human beings. The third affordance is the capacity of the Internet to support, extend and expand communications capabilities of humans in multiple formats across the bounds of time and space [6].

The Semantic Web based Education systems has become a necessity due the following key problems.

- Heterogeneity: The operational systems are heterogonous.

- Diverse Representations: There exists different representation of resources which are in the form of courseware, cartoon, video, audio, text, software and database. The metadata for these resources are different when built by different people. Thus making it difficult to organize and manage the resources. The reusability factor is low and the construction is at low level. Hence there is wastage of human and material resources.

- Limited Connectivity: There is lack in connectivity for related resources on a particular domain. Educational resources are used to introduce knowledge and knowledge segments are related by abundant semantic association. For example a user wants resources about 'AI', the systems usually reply only if the resource contains the word AI. However if the document does not contain the word AI and some other word like machine learning, the response would be nil.

- Low degree of self directed learning: The present elearning systems focus on showing knowledge to users rather than links among knowledge. This makes it difficult to regroup knowledge to increase or rebuild learner's knowledge systems. Thus it does not help in self directed learning.

- Low adaptability: The designers of the educational systems want the computer to be a tutor and a helper. Most systems concentrate on arranging unified content but rarely consider the differences of individual characteristics. This reduces the learning interests and efficiency reduces.

\section{SEMANTIC WEB}

The Semantic web tries to reform the existing web by adding in a layer that will enable advanced automatic processing of the web content so that data can be shared and processed by both humans and software. In simple words told[1], it is to try make the computers understand the web content to help the users with relevant information, make inferences and calculations from the information and combine information in new ways to support knowledge based tasks such as authoring, planning, navigation, cultural exchange and research. Some of the technologies [required for building the SWEBS are as follows:

- UML: UML is used to provide a collection of models and graphs to describe the structural and behavioral semantics of any complex information system. Some of the models[2] provided are

1. Use case models and scenario's to capture the user requirements and functionality of the system. Scenarios are instances of use cases

2. Class and object diagrams to specify the semantic information structure of a system.

3. Object diagrams are instances of class diagrams.

4. Activity diagrams to specify work flows. 
5. State diagrams to describe the dynamic behavior of an object in a system.

6. Interaction diagrams (sequence and collaboration diagrams) to model how groups of objects collaborate in some behavior.

7. Physical diagrams (deployment and component diagrams) to model the implementation structure of a system.

- XML and XML Schema's: HTML is more layout oriented but XML is more structure oriented. XML is user defined and semantics can be added to it. XML Schema provides the necessary framework for creating XML documents by specifying the valid structure, constraints, and the number of occurrences of specific elements, default values, and data types to be used in the corresponding XML documents. Schema vocabularies can be of a semantic nature and be used to support a culture of open exchange of data within the communities and tools that understand the vocabulary.

- RDF and RDF-Schema: This helps define the semantic meaning of data (metadata) on the web. It is done by specifying the O-A-V triplets or statements which is essentially a relation between an Object (Resource), Attribute (property) and a value (resource or free text). The O-A-V triplets are the semantic units for representing a domain. RDF Schema (RDFS) defines the vocabulary of an RDF model. It provides a mechanism to define domain-specific properties and classes of resources to which those properties can be applied, using a set of basic modeling primitives (class, subclass-of, property, sub property-of, domain, range, type). URI's are used to identify web resources and Namespaces with different vocabularies.

- $\quad$ Topic Maps: Topic maps (TM) represent information using topics, associations and occurrences. They are very similar to RDF, however some major differences exist. TM provides a higher level of semantic abstraction and allows n-ary relationship between any numbers of nodes whereas RDF is limited to triplets.

- Ontology: This is defined as text based knowledge representation so that the agents of the Semantic web can access this knowledge when necessary. Several languages exist for representing ontologies. One of them is OWL (Web Ontology Language), W3C officially released language. The increasing popularity of OWL might lead to its widest adoption as the standard ontology representation. OWL has basically three sublanguages- OWLDL, OWL Lite and OWL Full. There also exist authoring tools, such as PROTÉGÉ -2000 and Ontoedit. The tools are used to develop new ontologies and modify existing ones. They let the author edit and develop ontologies by concentrating on the domain's concepts and relationships, without having knowledge of the ontology-representation languages. The author can choose ontologies from a list, choose attributes and relations from another list, edit, add, remove, and merge ontologies. The output is produced will be in a specific high-level ontology-representation language such as OWL, in $\mathrm{RDF} / \mathrm{RDFS}$, in HTML, or in plain text.

- Software Agents: The definition of Software agents according to scholars is defined as a piece of software that can act proactively, adaptive and ability to communicate with other agents and its human users.
The above technologies can be classified into two divisions: Hard Semantic Technologies and Soft Semantic Technologies. Hard semantic technologies provide ways to express meanings of resources, their relationships in machine process able formats, and ways to draw conclusions, to reason based on these meanings. Examples include the Resource Description Framework (RDF), the Simple Knowledge Organization System (SKOS), and triple stores. Soft semantic technologies provide ways to express the meanings of resources in formats that humans can interpret, or in formats that employ domain-specific information structures. Examples include traditional tagging tools, topic maps, and domain-specific XML schemas.

\section{SEMANTIC WEB BASED EDUCATION SYSTEM}

Intelligent web based education system have been doing its round for several years. However Intelligence of a Web-based educational system means the capability of demonstrating some form of knowledge-based reasoning in various activities related to curriculum sequencing, in analysis of the student's solutions, and in providing interactive problem-solving support (possibly example-based) to the student, all adapted to the Web technology[4] .

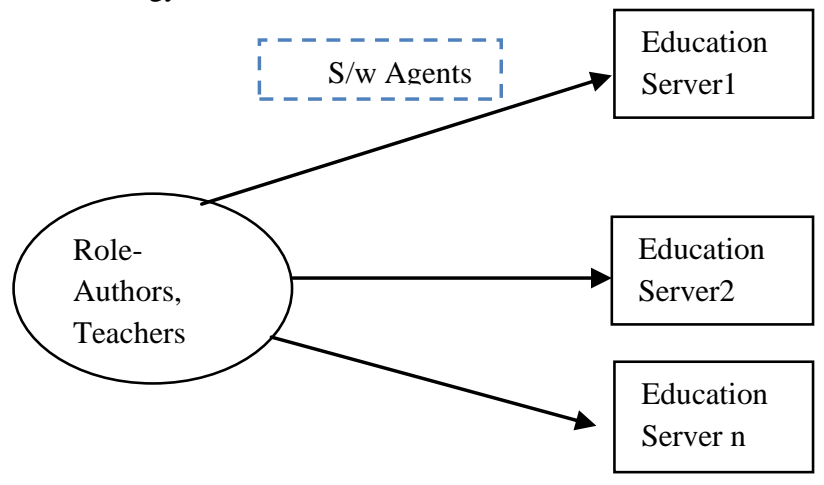

Fig 1: Reference Model for SWEBS

- Teacher's role, also called as content providers, help provide learning content and design learning activities. Its job is also to monitor learners' interactions (problem solving, assessment, etc.) and to configure learners' strategies, to support students' evaluation etc.

- Learner's Role: The main interest of learners is to interact with the system in order to receive personalized educational content in order to improve their knowledge and fulfill their learning goals;

- Author's Role: Authors are responsible for structuring the educational content. Authoring activities are concerned with (i) educational content, (ii) instructional process, and (iii) adaptation and personalization.

The pedagogical agent job is to access the educational content on the server placed at different locations by using high-level educational services. The educational server possesses enough intelligence to arrange for personalization of the learning tasks it supports. The server appears to act as an intelligent tutor with both domain and pedagogical knowledge to conduct a learning 
session from the learner's point of view. It uses a presentation planner to select, prepare, and adapt the domain material to show to the student. During interaction, the SWEBS gradually builds the student model during the session, in order to keep track of the student's actions, learning progress, detect and correct his/her errors and misconceptions. This helps redirect the session accordingly if required. The end result of the architecture is that the student need not perform the discovery of the relevant material manually.

\subsection{Ontology Model for SWEBS}

Providing semantic rich environments is one essential issue in current education system. For this, Ontologies make the backbone of the SWEBS. Ontology can be used into particularly two areas in the field of education i.e.to construct domain knowledge and instructional knowledge. The Semantic web vision mainly depends on the domain ontology being constructed from the web content which is understandable by the software agents[9]. Generating ontology and populating ontology are two different research areas. To complete the picture of Semantic web Based Education System, there are several issues to be addressed.

- Domain ontology built from text data.

- What Knowledge to be extracted?

- What approach is required for constructing educational ontology that is different from ontology constructed for other domains?

\subsubsection{Ontology Extraction from text}

The Ontology life cycle can be consolidated from paper [4] into four phases: the specification, the formalization, and maintenance and evaluation stage.

- $\quad$ Specification: Identification of the purpose and the scope of technology. This mainly depends on the domain area.

- Formalization: This phase is to produce the conceptual and formal model that satisfies the domain area.

- Maintenance: Allows Ontology update and evolution. Consistency of the ontology is also checked here.

- Evaluation Stage: Analyses the resulting ontology to check if it satisfies the initial needs and if it has the desired features.

For the above ontology engineering, it is best to use the authoring tool or ontology editor that can help formulate knowledge by construction of ontology. The ontology learning techniques can basically vary with the degree of automation, the ontological knowledge that has to be extracted, the knowledge sources and the purpose. The degree of automation may vary as semi automatic or automatic [9]. The ontological knowledge that has to be extracted from structured, semi structured or unstructured data include concepts, taxonomy, conceptual relationships, attributes instances and axioms. Construction of ontology can begin form scratch or by updating the existing ontology.

The first step to create ontology from text is to prepare a corpus related to domain interest. This corpus must be carefully chosen which adequately describes the domain. A number of subtasks have then been performed in order to learn domain ontology. Few of the learning tools that encompasses each task include Text-2-Onto, TEXCOMON (Zouaq and Nkambou, 2009a) (Zouaq and Nkambou, 2009b), OntoLearn [11] (Velardi et al., 2005), and OntoGen.

Basically the ontology constructed for the basis of education can be in support of different roles. The two main ontologies to be constructed are for the subject ontology and learner's ontology. The subject ontology of educational resources defines the subject's taxonomic relationship. When searching educational resources, the system can deduce the content concepts based on the subject classification system and get high quality resources. The learning ontology refers to the course system and teaching mechanism, which is used to describe the association between subjects, the knowledge points and the sequence of learning. In order to meet the demands of the autonomous learning system, various other ontologies as in paper [7] can be added such as the need surfaces. The process of feedback automatically and building ontology for the learning forum can be modeled into the system. From a learner perspective, in the learning forum, the ontology takes effect as the following:

- Describing the content of each reply,

- Imposing a structure on the information in terms of concepts,

- Retrieving information from ontology base,

- An important tool for resolving semantic heterogeneity problems,

- Providing a platform where replies can be annotated based on ontology

The subject course as described in [6] can be organized into the learning contents by dividing into the different chapters, where each chapter will have one or more knowledge points. The common terms are to be extracted from the basic contents whose various relationships constitute the semantic model or the ontology model. The ontology model becomes the foundation on which most of the important functions are realized. A smart navigation can consider the individual characters and needs of the learners. The flow can be as below:

1) Learners ask questions to be answered immediately.

2) An intelligent answer-question system can supply answer through the intelligent tutor (Teachers, Authors and Learners) or through agent to the learners as follow :

1) The students raise questions in this system

2) Teachers, authors and learners can answer

3) When the teachers are not online, a classical questions library can be used to store those that students always raise.

4) Learners ask some questions, the system can do semantic analysis for these questions and extract the core concepts.

5) System then compares these concepts with the concepts of the questions in the classic question library.

6) If the library has found out the answers of the related questions then display to learner 
7) Else the knowledge points from the knowledge-concept relation pool (ontology) according to the terms can be extracted.

3) The autonomous evaluation system can also provide the learners with a convenient environment to let them check anytime the content that has been mastered and the content to be learned.

In conclusion, in the context of SW and education [7], ontology can be applied to solve a variety of complex problems, such as knowledge representation in intelligent systems, knowledge sharing and reuse among applications, annotation and search of learning objects, personalization of learning content, beside many other challenges.

\subsubsection{Knowledge to be extracted}

Educational knowledge basically can be divided into two formsdeclarative and procedural knowledge. Researchers however focus on declarative knowledge which represents the actual and factual information for construction of ontology. The procedural knowledge is best represented by rule set formation which mainly contains the problem solving procedures and action sequences. The main objective behind these representations is to enable the system to possess a structured knowledge, to facilitate reasoning and to be able to provide the best adapted learning session to a learner.

Procedural knowledge has been best described in expert systems using heuristic rules. These kinds of rules are intuitive and easy to understand by a domain expert. However, with the growing number of rules, the inference process may not be efficient. Knowledge acquisition is another bottleneck of the approach, since it is very difficult to learn this kind of expertise without relying too heavily on domain experts.

Finally, rules necessitate a careful planning of the input and cannot handle unknown or incomplete inputs. Case-based reasoning (CBR) can represent a solution to the problem of handling unknown input. One of the strengths [10] of this approach is that it is capable of providing a conclusion even for unknown cases due to a similarity function.

\subsection{Approaches for knowledge representation}

Some of the knowledge representation form as discussed in paper [8] like concept maps, topic maps, and conceptual graphs represent knowledge in the form of a graphs composed of nodes (concepts) and links between nodes, which model the conceptual relationships between the concepts. A conceptual map is a technique (strategy, tool or resource) to represent and organize knowledge using concepts and linking phrases between these concepts. On the other hand, Topic Maps is an international rule to express in a computer readable format concept (topics), relationships between information concepts and resources linked to these concepts. A conceptual map is a group of propositions on a certain subject ordered in the shape of a tree. In the nodes of the tree the concepts are placed and in the nodes connections the linking phrases would be placed expressing the relationship between the connected concepts. Usually the concepts are the substantive part of the sentences, and the verbs or prepositions make up the linking phrases. There is always an initial concept (root) from which the relationship tree develops. The concepts are usually represented within boxes or ovals and the linking phrases act as labels of the lines linking one or more of these concept boxes.

When ontology is to be created for a subject, the main steps include the Glossary development which is used to gather all the information relevant to the described domain. Selecting and verbalization of all essential objects and concepts in the domain chosen is to be completed. The next phase is the Laddering where the main levels of abstraction are defined. High Level Hierarchy is to be constructed by associating similar concepts to create meta-concepts from leaves of the aforementioned hierarchy. The third step is the disintegration. The objective of this step is breaking of high level concepts into a set of detailed ones where it is needed. This could be done via a top-down strategy trying to split the high level concept from the root of previously built hierarchy. The last step forms the refinement step. This is devoted to updating the visual structure by excluding the excessiveness, synonyms and contradictions. The ultimate goal of the final step is to create a complete, reusable and updatable ontology.

\section{AN ARCHITECTURE FOR SWEBS}

The paper [3] discusses architecture shown in Figure 2 for Semantic Web Education Based System. It was basically created for a training institute. However the architecture does form the basis for the development of Semantic Web Based Education System. The authors have used RDF, OWL and Semantic Web Mining to develop an enterprise web framework. The database contains information regarding the users and the access right policies. The ontology has been created for the Teacher, Author and Learner role. Ontology has also been created for the user's access policy and security policy solution table.

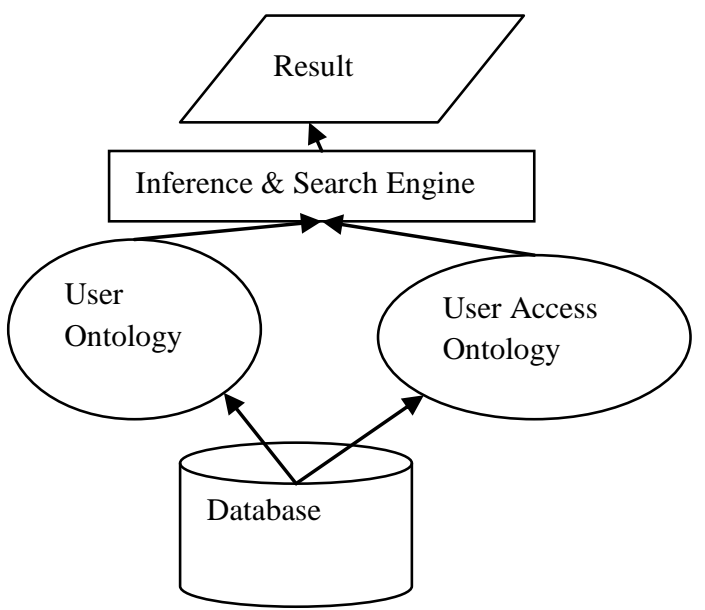

Figure 2: Architecture for SWEBS

The knowledge repositories are the corpus available as the communal database and World Wide Web which are used to help find data with the students, faculties and courses. Results from the reference and Reasoning Engine can be given to the learners. The architecture can also benefit from a mental model composed by attributes and preferences of the student which can be set in a module called student model (SM). Such SM also includes an engine devoted to carry out inferences about the behavior and the learning of the student. 


\section{CONCLUSION}

The Semantic Web has a big potential for improving technology-enhanced learning in many respects. The expectation is that the SW-based educational applications will enable the realization of AAAL: Anytime, Anywhere, Anybody Learning using most of the WWW resources as reusable learning objects supported by standard technologies. This trend facilitates the use of WWW content and resources as knowledge that can be interpreted by computers and learned by people. This paper tries to cover an introduction to the Semantic web layer cake with respect to Education System. The Semantic Web Based Education System architecture has been discussed upon. Various possible ontology and the steps of construction on a particular domain also has been discussed to complete the picture of Semantic web based Education System.

\section{REFERENCES}

[1] E Liu, M Xu and Z Chai, "Research on the Autonomous Learning System Based on Ontology Model", First International Workshop on Education Technology and Computer Science, 2009.

[2] V.Devedzic,"Education and the Semantic Web", International Journal of Artificial Intelligence in Education 14 (2004) 39-65

[3] A.Nayak, J Agarwal, "Enterprise Architecture for Semantic Web Mining in Education", Second International Conference on Computer and Electrical Engineering, IEEE pp 23-26, 2009.

[4] Gómez-Pérez, A., \& Corcho, O. (2002). Ontology Languages for the Semantic Web. IEEE Intelligent
[5] Koper R.," Use of the Semantic Web to Solve Some Basic Problems in Education:"Workload. Journal of Interactive Media in Education, 2004 (6).

[6] Corcho, O.; Fernández-López, M. and Gómez-Pérez, A. 2007. Ontology engineering: what are ontologies and how can we build them, in Cardoso, J. (Ed.). Semantic Web: Theory, Tools and Applications, Information Science Reference London 44-70

[7] G.Ivanovic, L Nimac and N Perse, "Towards Semantic Web Based Web application for Academic Programs in Croatia, Proceedings of the ITI 2010 32nd Int. Conf. on Information Technology Interfaces, June 21-24, 2010, Cavtat, Croatia

[8] Gavril ova T. Ontological Engineering for Practical Knowledge Work. Lecture Notes in Artificial Intelligence 4693, Proc. of 11-th Int. Conf. "Knowledge-Based Intelligent Information and Engineering Systems KES 2007", Italy.

[9] F F M Ghaleb, S S Dhaoud and A Hasna, "A Web Based E-Learning System using Semantic Web Framework", Journal of Computer Science 2 (8): 619-626, 2006 ISSN 1549-3636,2006 Science Publications

[10] A Zouaq and R Nkambou,"Building Domain Ontology from Text for Educational purposes", IEEE Transactions on Learning technologies, Vol 1, No 1, January-March 2008

[11] Velardi, P., Navigli, R., Cuchiarelli, A.and Neri, F. (2005). Evaluation of ontolearn, a methodology for automatic population of domain ontologies. In P. Buitelaar, P. Cimiano, and B. Magnini, editors, Ontology Learning from Text: Methods, Applications and Evaluation. IOS Press. 